\title{
Scheduling algorithms for advance resource reservation
}

\author{
Charlie $X u$ and J.W. Wong \\ Department of Computer Science, University of Waterloo \\ Waterloo, Ontario, Canada N2L $3 G 1$ \\ Telephone: 519-885-1211, Fax: 519-885-1208 \\ e-mail: jwwong@bcr.uwaterloo.ca
}

\begin{abstract}
Advances in high performance networking have provided improved support to resource intensive applications, e.g., video conferencing and video-on-demand. For conferencing applications, the ability to reserve network resources in advance is highly desired. Most previous works on advance resource reservation are based on a single-link model. We extend this model to a network environment with emphasis placed on multi-party conferencing. A reservation request is characterized by its start time, bandwidth requirement, holding time, and participating parties. Both immediate and delayed acknowledgments are considered. In immediate acknowledgment, the response to a reservation request is returned as soon as possible. In delayed acknowledgment, requests are batched for scheduling purposes with a view that the system may be able to make more informed decisions. However, the delay may be unacceptable to the requester. In this paper, scheduling heuristics are developed for delayed acknowledgment. Simulation results on the performance difference of these heuristics and immediate acknowledgment are presented.
\end{abstract}

\section{Keywords}

Resource management, advance reservation, scheduling

\section{INTRODUCTION}

Traditionally network services, such as file transfer and remote access to databases, generally operate on an "on-demand" principle. This means that the user submits a request for service at the very moment that he/she wishes the communication to take place. Most studies on network resource management are based on this scenario. Advances in high performance networking have provided improved support to resource-intensive applications, e.g., video conferencing and video on demand. These applications invariably require some quality of service (QoS) guarantees from the network to ensure efficient operation. Much research has been done, and active research is underway, on 
the various aspects of a communication network that supports QoS guarantees. A key assumption is again the on-demand nature of service requests. However, for conferencing applications, the need for successful call setup at a pre-specified time argues for advance resource reservation (ARR), i.e., resource reservation made some time before the actual setup of a call. The need for this option in high-speed networks is mentioned in CCITT's Recommendation I.121 on broadband aspects of ISDN [1] and in IETF's Internet draft on session initiation protocol [2].

Performance investigation of ARR systems have been reported in [3-10]. These studies are concerned with the design and evaluation of scheduling algorithms for ARR using single link models. In other studies, architectural issues of ARR systems [11] and mechanisms for establishing a reservation [12] have been investigated. In this paper, we extend previous works on single-link models to a network environment. Our focus is on multi-party communication since it is likely to be the main user of ARR systems. Moving to a network environment introduces new research challenges in network resource management such as scheduling and routing of calls reserved in advance.

A number of network resources can be considered as candidates for advance reservation. Examples are bandwidth and buffer space. For simplicity, we only consider bandwidth reservation, which is the dominant factor in QoS guarantees. The amount of bandwidth required is specified in a reservation request. For example, voice traffic would require $64 \mathrm{~Kb} / \mathrm{s}$ or less, depending on the encoding algorithm used. Video may also be transmitted at a range of data rates, depending on the encoding algorithm and the quality level.

A important consideration in ARR is the acknowledgment delay, which is the elapsed time from when a reservation request is made to when the result of the request is known. From the perspective of the requester, the acknowledgment delay should be kept to a minimum (referred to as immediate acknowledgment). However, by batching requests, an ARR system may be able to make more informed scheduling decisions, and utilize network resources more efficiently. Hence, there may be an advantage in delaying scheduling decisions, and thereby delaying the acknowledgments. Of particular interest to this investigation is the performance benefits of delayed acknowledgment over immediate acknowledgment.

In general, exact analytic results for the performance of reservation systems are difficult to obtain, even for single-link models. Most investigations rely on the use of simplified models, approximate analysis, or simulation. Network-scale models are even more complex. For example, routing of multipoint connections is known to be NP-hard except for some specific network topologies [13]. Our investigation is based on simulation. We first develop scheduling heuristics for delayed acknowledgment, and then evaluate the performance difference between these heuristics and immediate acknowledgment.

This paper is organized as follows. Section 2 defines the performance model and the performance metrics used in our investigation. Our scheduling heuris- 
tics are developed in section 3. In section 4, results on performance comparison of immediate acknowledgment and delayed acknowledgment are presented. Finally, section 5 contains a summary of our findings.

\section{PERFORMANCE MODEL}

In general a complete model of an ARR system contains the following components: the network model, the request characteristics, and the reservation model. These components, as well as the performance metrics, are defined in this section.

\subsection{Network model}

The network under consideration consists of a set of switches connected by communication links and is modeled by a connected graph $G=(V, E, w)$, where $V$ is a set of nodes representing the switches, $E$ is a set of edges representing the communication links, and $w: E \rightarrow R$ is a link cost function. It is assumed that the capacity, or bandwidth, of a link is organized into $N$ fixed-sized bandwidth units. For example, with $1 \mathrm{~Kb} / \mathrm{s}$ units in a $1.5 \mathrm{Mbps}$ channel, $N$ is 1500 . A request may only reserve a multiple of these units. On a given link, the difference between the total capacity and the reserved capacity is the amount of capacity still available for reservation or other services. This amount of capacity is called the residual capacity.

In our simulation experiments, we use random graphs with 100 nodes as our sample networks. The average degree of a node (number of links adjacent to a node) is 3 . The random graphs are generated using the Stanford GraphBase package [14].

\subsection{Request characteristics}

In general, each party in a multi-party conference may need different amount of bandwidth for sending and receiving data. For example, a party has one outgoing video/audio stream but may have to receive several incoming streams. This asymmetry may result in a network with asymmetric residual capacities on the links. Routing a multi-party connection with asymmetric capacity requirements is a very hard problem. A simplification to this problem is the routing of a point-to-multipoint connection where one of the parties is the source and the other parties just listen. This is the Steiner tree problem in directed networks (STDN). Good approximation techniques are not available for this problem. Recently two groups of authors obtained non-trivial approximation algorithms for STDN $[15,16]$. Their algorithms achieve an approxi- 
mation ratio of $i(i-1) k^{1 / i}$ in time $O\left(n^{i} k^{2 i}\right)$ for any fixed $i>1$, where $k$ is the number of parties. For $i=\log k$, they obtain $O\left(\log ^{2} k\right)$ approximation ratio in quasi-polynomial time.

To reduce complexity, we assume that for a given reservation, the capacity requirement on both directions of each link is the same. We represent a call request by a 4 -tuple $(c, s, h, p)$, where $c$ is the capacity requirement; $s$ is the start time; $h$ is the holding time; and $p \subseteq V$ is the set of parties. Each request, if accepted, will result in reservations along a tree spanning all the parties. The same capacity $c$ is reserved in both directions on each link of this tree. This is called the uniform capacity assumption.

For convenience, we assume, as in [3], that time on each link is divided into fixed length units called slots. At most one request may arrive in a slot and such an arrival occurs with probability $\lambda$. The start time of a request can only be at the beginning of a slot and the holding time is an integral multiple of slots.

\subsection{Reservation model}

The timing diagram in Figure 1 shows how a call request is handled $[3,11]$. This request is submitted at time $t_{\text {req }}$, and an acknowledgment is returned at time $t_{\text {ack. }}$. At the requested start time $t_{\text {begin }}$, the reserved resources are allocated and the conference begins. The difference between $t_{\text {req }}$ and $t_{\text {begin }}$ is called the notice interval.

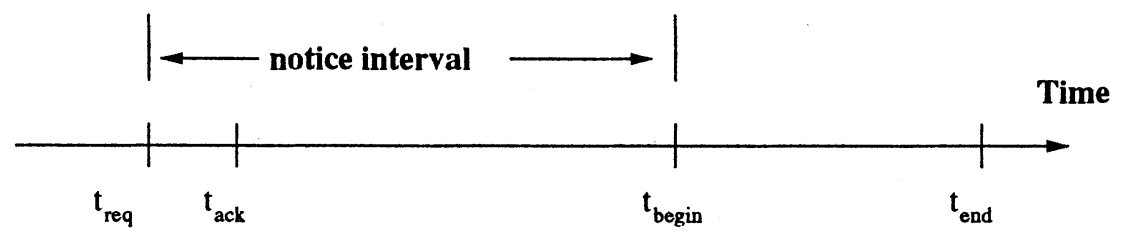

Figure 1 Timing diagram for request handling

Because the available bandwidth is finite, it is not always possible to accommodate all call requests. One approach to handling conflicts is to find an alternative start time, preferably close to the requested start time. Another approach is to reject any requests that cannot be accommodated. It is up to the requester to submit another request for an alternative start time. This is often referred to as a loss system. This paper is concerned with loss systems only.

As mentioned in the introduction section, reservation requests can be handled using immediate acknowledgment or delayed acknowledgment. Modeling of immediate acknowledgment is straight-forward. Each time a request is submitted, a scheduling decision is made immediately. 
For delayed acknowledgment, the model in [3] is adopted (see Figure 2). Time on each link is partitioned into reservation periods of length $Y$ slots. A decision point occurs at the start of each reservation period. When a decision point is reached, all requests associated with that decision point are scheduled. For a given request, its associated decision point is characterized by a global parameter $P$, called the decision point offset. Consider the arrival of request $k$. The earliest possible decision point $E_{k}$ is the first decision point after the arrival. The latest possible decision point $L_{k}$ is the latest decision point before the requested start time. In this model, the decision point for request $k$ is given by $\min \left(L_{k}, E_{k}+P\right)$. When $P=0$, the system behavior is similar to that of the immediate acknowledgment model. Increasing $P$ has the effect of delaying the scheduling decision. If $P$ is so large that all the requests are scheduled at their latest possible decision point, the behavior is similar to that of a system where batching of reservation requests is maximized.

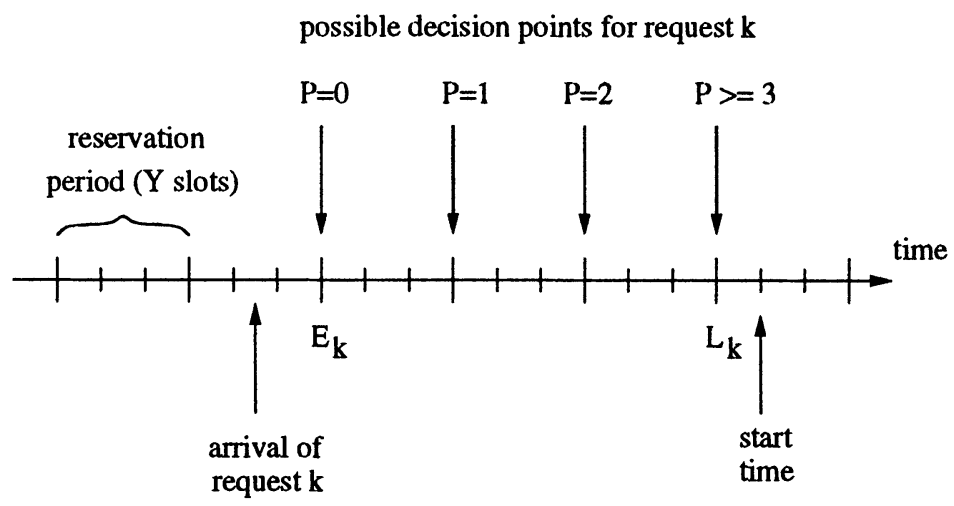

Figure 2 The delayed acknowledgment model

\subsection{Performance metrics}

In previous works on single-link models, performance metrics such as blocking probability and channel utilization were used. The extension of ARR to a network environment introduces the need for additional performance metrics.

In this study, we consider blocking probability and two other performance metrics. The first is related to load balancing. It may be desired that the reserved capacity on the links be balanced so as to better serve traffic classes other than advance reservation, e.g., on-demand requests. The variance of residual capacities is used as our performance metric. Suppose the residual capacity of a link $e$ at time $t$ is $r_{e}(t)$, the variance of residual capacities of the 
network $G$ at time $t$ is defined as

$\sigma_{G}^{2}(t)=\frac{\sum_{e \in E}\left(r_{e}(t)-\bar{r}_{G}(t)\right)^{2}}{|E|}$

where $|E|$ is the number of links and $\bar{r}_{G}(t)$ is the average residual capacity of the network at time $t$ over all the links:

$\bar{r}_{G}(t)=\left(\sum_{e \in E} r_{e}(t)\right) /|E|$

Over a period of time from $t_{0}$ to $t_{1}, t_{1}>t_{0}$, we define the time-averaged variance of residual capacities as

$\bar{\sigma}_{G}^{2}\left(t_{0}, t_{1}\right)=\frac{1}{t_{1}-t_{0}} \int_{t_{0}}^{t_{1}} \sigma_{G}^{2}(t) d t$

The second performance metric is the total reserved capacity. It is defined as the sum of reserved bandwidth on each link in the network. Let $b_{e}(t)$ be the amount of the bandwidth reserved at link $e$, the total reserved capacity in $G$ at time $t$ is

$$
C_{G}(t)=\sum_{e \in E} b_{e}(t)
$$

The time-averaged total reserved capacity in network $G$ from time $t_{0}$ to $t_{1}$, $t_{1}>t_{0}$, is

$\bar{C}_{G}\left(t_{0}, t_{1}\right)=\frac{1}{t_{1}-t_{0}} \int_{t_{0}}^{t_{1}} C_{G}(t)$

These above performance metrics implicitly assume that all links are equal in their importance. In our simulations, we experiment with several networks and average the results. We will therefore drop the subscript $G$ in our discussions.

\section{SCHEDULING HEURISTICS}

\subsection{The scheduling problem}

In general, the scheduling problem in an ARR system can be described as follows. Consider a list of one or more reservation requests $R_{1}, R_{2}, \ldots, R_{q}$, where $R_{i}=\left(c_{i}, s_{i}, h_{i}, p_{i}\right)$. The first step is to establish an order in which 
these requests are to be processed. The next step is to perform admission control and routing for each request on the list, according to the established order. Note that for immediate acknowledgment, the list always contains one request, and for delayed acknowledgment, the list corresponds to requests that are associated with a decision point.

For admission control, a request $R_{i}$ is accepted if the network has sufficient capacity to support it, otherwise it is rejected (or blocked). Routing is then performed for the accepted request. This involves the determination of a tree that spans the participating parties. In our study, we use the minimum spanning tree (MST) heuristic [17] to construct the tree. This heuristic is relatively easy to implement. It achieves a tight performance ratio of $2-2 / k$ with overall worst-case time complexity $O\left(k n^{2}\right)$, where $k$ is the number of parties, and $n$ is the number of nodes in the network. In practice, the average performance of MST heuristic is much better than its worst bound.

The MST heuristic uses a link cost function for tree construction. Our cost function is based on an instantaneous link cost that is exponential in the utilization of that link. The instantaneous cost of a link $e$ at time $t$ is defined as:

$w_{e}(t)=\delta+\mu^{\frac{b_{e}(t)}{N_{e}}}-1, \quad \mu>1$

where $\mu$ is a chosen parameter, $N_{e}$ is the total capacity of link $e, b_{e}(t)$ is the reserved capacity of link $e$ at time $t$, and $\delta$ is a small constant. The reason for the presence of $\delta$ is that it prevents the cost of a link from dropping to zero when no reservation is made on that link. The cost function used by the MST heuristic is the average cost over the holding time of the request, say from $t_{0}$ to $t_{1}$. This average cost at link $e$ is given by

$\bar{w}_{e}\left(t_{0}, t_{1}\right)=\frac{1}{t_{1}-t_{0}} \int_{t_{0}}^{t_{1}} w_{e}(t) d t$

The choice of $\mu$ is discussed in [19]. It is found that as $\mu$ increases, the load is more balanced at the expense of higher resource usage. It is also found that performance is not satisfactory when $\mu$ is too large. In this investigation, we choose $\mu=2.0$.

\subsection{Heuristics}

For delayed acknowledgment, we need a heuristic to order the requests that are to be scheduled at each decision point. Of interest are heuristics that are based on total resource usage. For our investigation, the total resource usage of request $R_{i}$ is estimated by $u_{i}$, which is calculated as follows: 
- Perform routing for $R_{i}$, assuming that all links in the network have full capacity, i.e., no advance reservation has been made. The result is a tree $T_{i}$.

- Calculate $u_{i}$ as the product of total reserved bandwidth and holding time. That is $u_{i}=\left(\sum_{e \in T_{i}} c_{i}\right) \cdot h_{i}$.

Based on this estimate, we define the following heuristics:

- Maximum estimated resource usage first (MAXEU) - the requests are ordered in decreasing order of $u_{i}$ 's.

- Minimum estimated resource usage first (MINEU) - the requests are ordered in increasing order of $u_{i}$ 's.

Intuitively, the MINEU heuristic may be a good choice for minimizing the blocking probability since requests that require less resources are given higher priority. The MAXEU heuristic may be a good choice for load balancing because requests with large resource usage are scheduled first, and requests with small resource usage are scheduled later to fine tune the balance.

\section{SIMULATION RESULTS}

\subsection{Preliminary observations}

As a first step to understand the performance difference of MAXEU and MINEU for delayed acknowledgment, we use a simplified model which would allow us to obtain some initial insight on load balancing. In this simplified model, all requests have the same start time and same holding time. Requests are scheduled as a batch. The model parameters are as follows:

- Capacity of each link - 1000 units,

- Capacity requirement of each request - uniform over the interval $[1,75]$,

- Number of participating parties - binomial with mean 30 ,

- Number of requests in the batch - binomial with mean $\bar{q}$.

We run the simulation on 10 randomly generated networks and average the results. The values of $\bar{q}$ are selected such that the blocking probabilities are negligible. Figure 3 shows the distributions of residual capacity in the network as a result of applying the MINEU and MAXEU heuristics. It is observed that MAXEU results in $29.9 \%$ fewer links in the low capacity region (with residual capacity $<700$ units) and $27.3 \%$ fewer links in the high capacity region (with residual capacity $>900$ units). The distributions show that the use of MAXEU can lead to a more balanced load. 


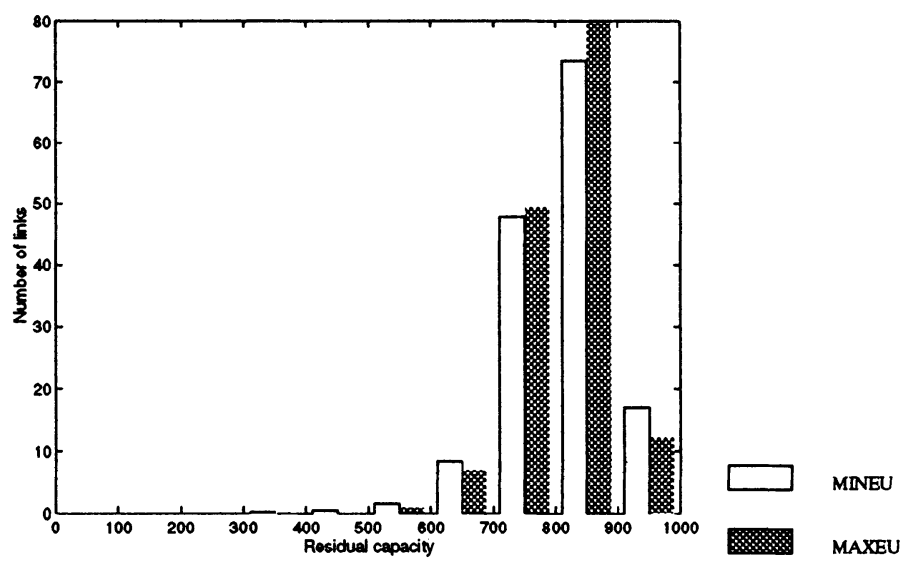

Figure 3 Simplified model: Distribution of residual capacity at $\bar{q}=15$

\subsection{Comparison between immediate and delayed acknowledgment}

We now evaluate the performance of the MAXEU and MINEU heuristics, when used in the delayed acknowledgment model. Of interest is their performance when compared to immediate acknowledgment (I-ACK).

In our delayed acknowledgment model, the notice interval is assumed to be uniformly distributed over the interval $[Y+1, T+Y]$. This ensures that no requests require service before the first decision point. We choose $T=480$, $Y=80$ and $P=6$. In this setting, all requests will be scheduled at the latest possible decision point since $P \geq\lceil T / Y\rceil$. The remaining model parameters are as follows:

- Capacity of each link - 1000 units,

- Capacity requirement of each request - uniform over the interval $[1,100]$,

- Number of participating parties - binomial with mean 40 ,

- Holding time - binomial with mean 20.

We study the performance of the scheduling heuristics as a function of the request arrival rate $\lambda$.

In our simulations, the parameters have been chosen to ensure small blocking probabilities. While a high blocking probability is acceptable in single-link models since it only causes the routing algorithm to choose alternative routes, it is hardly acceptable in a network model. Future networks are most likely to be designed with sufficient resources to ensure small blocking probabilities.

Simulation results for the blocking probabilities for selected values of $\lambda$ are shown in Table 1. We observed no blocking for $\lambda<0.30$. For larger values of 
Table 1 Blocking probability (\%)

\begin{tabular}{cccc}
\hline$\lambda$ & I-ACK & MAXEU & MINEU \\
\hline 0.30 & 0.0010 & 0.0010 & 0.0010 \\
\hline 0.35 & 0.0096 & 0.0154 & 0.0069 \\
\hline 0.40 & 0.0125 & 0.0182 & 0.0089 \\
\hline
\end{tabular}

$\lambda(\lambda=0.35$ or 0.40$)$ the MINEU and MAXEU heuristics yield the smallest and largest blocking probabilities, respectively. We conclude that for I-ACK, MINEU, or MAXEU, the blocking probability is negligible as long as the arrival rate does not exceed some specified value. Among the three algorithms, MINEU tends to yield the lowest blocking probability for a given arrival rate.

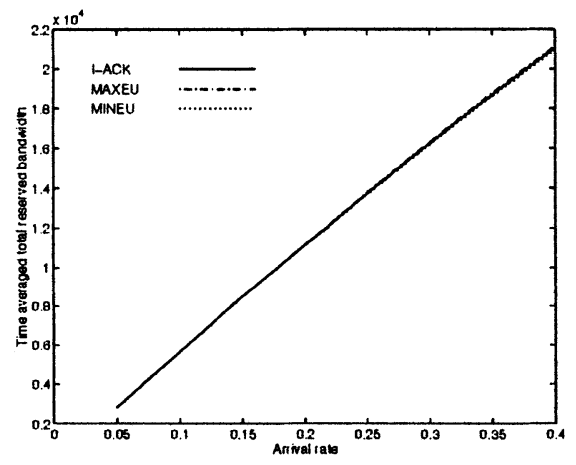

(a) $\bar{C}$ as a function of $\lambda$

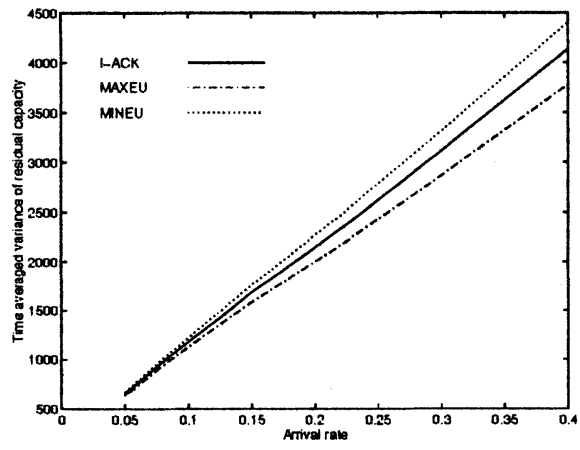

(b) $\bar{\sigma}^{2}$ as a function of $\lambda$

Figure 4 Performance comparison of immediate and delayed acknowledgment

Let $\bar{C}$ and $\bar{\sigma}^{2}$ be, respectively, the total reserved capacity and variance of residual capacity averaged over the entire simulation. $\bar{C}$ and $\bar{\sigma}^{2}$ are given by Equation (2) and (4) where $t_{0}$ and $t_{1}$ correspond to the start and end of simulation. In Figure $4 a, \bar{C}$ is plotted against $\lambda$. We observe that there is little difference among the heuristics with respect to resource usage. Figure $4 \mathrm{~b}$ shows the corresponding plot for $\bar{\sigma}^{2}$. We observe that MAXEU results in a smaller variance than I-ACK while MINEU results in a larger variance. The performance differences are noticeable, but not significant.

To gain further insight into resource usage and load balancing, we consider the total reserved capacity and variance of residual capacity averaged over 
non-overlapping periods of 500 slots. The arrival rate $\lambda=0.40$. Figure $5 \mathrm{a}$ shows the values of

$$
\bar{C}_{G}(500 i, 500(i+1)) \text { for I-ACK }-\bar{C}_{G}(500 i, 500(i+1)) \text { for MAXEU }
$$

and Figure $5 \mathrm{~b}$ shows the values of

$$
\bar{\sigma}_{G}^{2}(500 i, 500(i+1)) \text { for I-ACK }-\bar{\sigma}_{G}^{2}(500 i, 500(i+1)) \text { for MAXEU }
$$

for $0 \leq i \leq 200$. We observed that for most of the time slots, MAXEU leads to slightly higher resource usage, but a smaller variance. Analogous results (not shown) are obtained for the difference between MINEU and I-ACK, namely, resource usage is similar, but MINEU has larger variance.

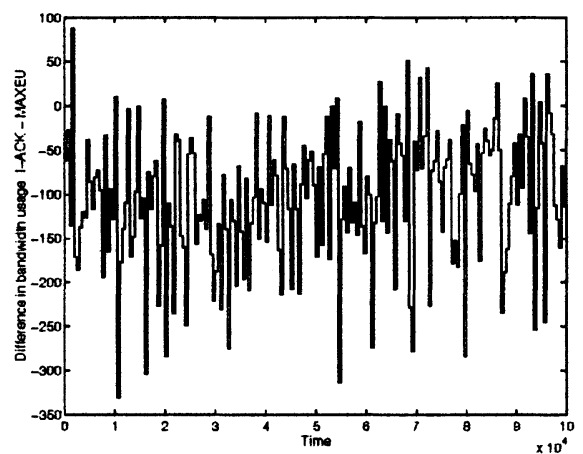

(a) Difference in $\bar{C}_{G}(500 i, 500(i+1))$

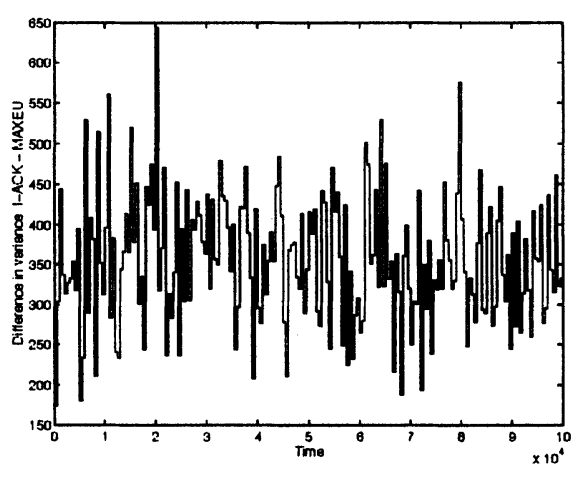

(b) Difference in $\bar{\sigma}_{G}^{2}(500 i, 500(i+1))$

Figure 5 Performance difference between I-ACK and MAXEU at $\lambda=0.40$

\section{CONCLUSION}

In conclusion, delayed acknowledgment with the MAXEU or MINEU heuristic is similar to I-ACK in terms of resource usage. MINEU yields the lowest blocking probability but does not perform as well with respect to load balancing. On the other hand, MAXEU is better in terms of load balancing, but has the highest blocking probability. Unless load balancing is an important issue, it seems that I-ACK is the best alternative, especially when one considers the fact that it provides immediate response to the requester.

\section{REFERENCES}

[1] CCITT (1989) Blue Book, Volume III, Fascicle III.7, Integrated Service 
Digital Network (ISDN) - General Structure and Service Capabilities, Recommendations I.110-I.257, ITU, Geneva.

[2] Handley, M., Schulzrine, H. and Schooler, E. (1997) SIP: session initiation protocol, Internet Draft, Internet Engineering Task Force.

[3] Harms, J.J. and Wong, J.W. (1995) Performance modeling of a channel reservation service. Computer Networks and ISDN Systems, 27, 148797.

[4] Virtamo, J.T. (1992) A model of reservation systems. IEEE Transactions on Communications, 40.

[5] Virtamo, J.T. and Aalto, S. (1991) Stochastic optimization of reservation systems. Eur. J. Oper. Res., 51, 327-37.

[6] Diaz Quinones, M.A. (1991) A simplified model of discrete-time reservation systems, in Proc. 13th International Teletraffic Congress, Copenhagen, Denmark, 647-52.

[7] Roberts, J.W. and Liao, K. (1986) A queueing model of an advanced reservation system with blocked requests lost, COST 214 Doc. 076.

[8] Liang, Y., Liao, K., Roberts, J.W. and Simonian, A. (1988) Queueing models for reserved set up telecommunications services, in Proc. 12th. International Teletraffic Congress, Torino, Italy, 4.4B.1.1-7.

[9] Reinhardt, W. (1994) Advance reservation of network resources for multimedia applications, in Proc. 2nd International Workshop, IWACA '94, Heidelberg, Germany.

[10] Degermark, M., Kohler, T., Pink, S. and Schelen, O. (1995) Advance reservations for predicted services, in Proc. 5th International Workshop, NOSSDAV '95, Durham, New Hampshire.

[11] Wolf, L.C., Delgrossi, L., Steinmetz, R., Schaller, S. and Wittig, H. (1995) Issues of reserving resources in advance, in Proc. 5th International Workshop, NOSSDAV '95, Durham, New Hampshire.

[12] Ferrari, D., Gupta, A. and Ventre, G. (1995) Distributed advance reservation of real-time connections, Proc. 5th International Workshop, NOSSDAV '95, Durham, New Hampshire.

[13] Winter, P. (1987) Steiner problem in networks: a survey, Networks, 17, 129-67.

[14] Knuth, D.E. (1994) The Stanford GraphBase : a platform for combinatorial computing. Addison-Wesley, New York, 384-97.

[15] Charikar, M., Chekuri, C., Goel, A. and Guha, S. (1997) Approximation algorithms for directed Steiner problems, Technical Report STAN-CSTN-97-56, Department of Computer Science, Stanford University.

[16] Cheung, T., Dai, Z. and Li, M. (1997) Approximating the Steiner problems on directed graphs, Technical Report TR-9\%-1, Department of Computer Science, City University of Hong Kong.

[17] Bharath-kumar, K. and Jaffe, J.M. (1983) Routing to Multiple Destinations in Computer Networks. IEEE Transactions on Communications, Com-31, 343-51. 
[18] Xu, C. (1998) Advance resource reservation networks, Master Thesis, Department of Computer Science, University of Waterloo.

\section{ACKNOWLEDGEMENT}

This work was supported by the Natural Sciences and Engineering Research Council of Canada.

\section{BIOGRAPHY}

Charlie $\mathrm{Xu}$ received the joint B.S. degree in computer science and mathematics from Simon Fraser University in 1996, and the M.Math degree in computer science from the University of Waterloo in 1998. His research interest is in the area of network resource management.

J.W. Wong received the B.S. degree in engineering, and the M.S. and Ph.D. degrees in computer science from the University of California at Los Angeles in 1970,1971 , and 1975, respectively. Since 1975, he has been with the University of Waterloo where he is currently a professor of computer science. From 1989 to 1994, he was Associate Provost, Computing and Information Systems. He was a visiting scientist at the IBM Zurich Research Laboratory from September 1981 to August 1982, from September 1988 to August 1989, and from September 1995 to August 1996. Dr. Wong was Editor for Wide Area Networks for the IEEE Transactions on Communications from 1989 to 1992, and served on the Editorial Board of Performance Evaluation from 1986 to 1993. He is currently on the Editorial Board of the IEEE/ACM Transactions on Networking. He was Technical Program Chair of IEEE INFOCOM '84 and of the 1994 International Conference on Computer Communications and Networks. His research interests include network resource management, distributed multimedia applications, and performance evaluation. 